\title{
The genome sequence of the garden bumblebee, Bombus
}

\section{hortorum (Linnaeus, 1761) [version 1; peer review: 3 approved]}

Liam M. Crowley (iD),

University of Oxford and Wytham Woods Genome Acquisition Lab,

Darwin Tree of Life Barcoding collective,

Wellcome Sanger Institute Tree of Life programme,

Wellcome Sanger Institute Scientific Operations: DNA Pipelines collective, Tree of Life Core Informatics collective, Darwin Tree of Life Consortium

${ }^{1}$ Department of Zoology, University of Oxford, Oxford, OX1 3SZ, UK

V1 First published: 14 Oct 2021, 6:270

https://doi.org/10.12688/wellcomeopenres.17187.1

Latest published: 14 Oct 2021, 6:270

https://doi.org/10.12688/wellcomeopenres.17187.1

\section{Abstract}

We present a genome assembly from an individual female Bombus hortorum (the garden bumblebee; Arthropoda; Insecta; Hymenoptera; Apidae). The genome sequence is 296 megabases in span. The majority of the assembly is scaffolded into 18 chromosomal pseudomolecules.

Keywords

Bombus hortorum, garden bumblebee, genome sequence, chromosomal

This article is included in the Tree of Life gateway.

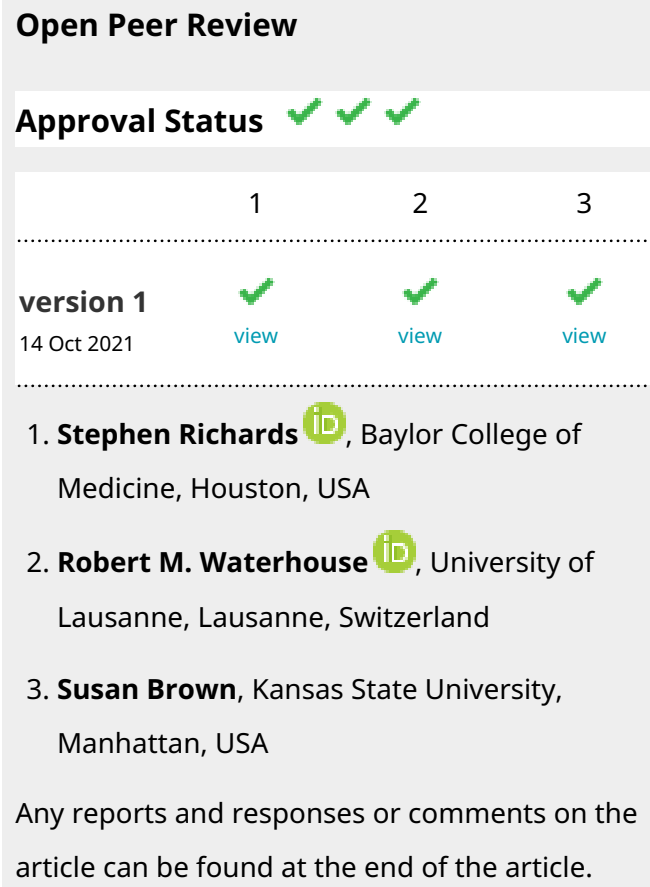


Corresponding author: Darwin Tree of Life Consortium (mark.blaxter@sanger.ac.uk)

Author roles: Crowley LM: Investigation, Methodology, Resources, Writing - Original Draft Preparation, Writing - Review \& Editing; Competing interests: No competing interests were disclosed.

Grant information: This work was supported by Wellcome through core funding to the Wellcome Sanger Institute (206194) and the Darwin Tree of Life Discretionary Award (218328).

The funders had no role in study design, data collection and analysis, decision to publish, or preparation of the manuscript.

Copyright: (c) 2021 Crowley LM et al. This is an open access article distributed under the terms of the Creative Commons Attribution License, which permits unrestricted use, distribution, and reproduction in any medium, provided the original work is properly cited.

How to cite this article: Crowley LM, University of Oxford and Wytham Woods Genome Acquisition Lab, Darwin Tree of Life Barcoding collective et al. The genome sequence of the garden bumblebee, Bombus hortorum (Linnaeus, 1761) [version 1; peer review: 3 approved] Wellcome Open Research 2021, 6:270 https://doi.org/10.12688/wellcomeopenres.17187.1

First published: 14 Oct 2021, 6:270 https://doi.org/10.12688/wellcomeopenres.17187.1 


\section{Species taxonomy}

Metazoa; Arthropoda; Insecta; Endopterygota; Hymenoptera; Apocrita; Aculeata; Apoidea; Apidae; Apinae; Bombini; Bombus; Megabombus; Bombus hortorum Linnaeus 1761 (NCBI:txid85660).

\section{Introduction}

The garden bumblebee, Bombus hortorum, is one of the seven most common species of bumblebee in the UK. It is widespread, being found in most habitats apart from upland areas. It is a large bumblebee species with a long face and a very long proboscis, meaning it favours deep flowers with a relatively long corolla such as foxglove (Digitalis purpurea) and honeysuckle (Lonicera periclymenum). It visits a wide range of flowers, particularly those with deep or complex blooms, meaning it is frequently found in gardens. This species expresses a preference for red clover (Trifolium pratense) when available (Brown et al., 1992). In common with other species in the Genus, the high degree of floral visitation undertaken by this species indicates its important role as a pollinator.

Bombus hortorum is a eusocial, annual species, with queens emerging from overwinter diapause from around March onwards. Workers can be seen from late April, with the number of workers increasing throughout the spring to reach around 100 workers in a mature nest (Edwards \& Jenner, 2005). The first males can be produced from June and new queens from July. Nests are always constructed under cover, but if underground they are often only shallowly so (Kells \& Goulson, 2003).

\section{Genome sequence report}

The genome was sequenced from a single female B. hortorum collected from Wytham Woods, Oxfordshire, UK (latitude 51.77, longitude -1.339). A total of 82-fold coverage in Pacific Biosciences single-molecule long reads and 113-fold coverage in 10X Genomics read clouds were generated. Primary assembly contigs were scaffolded with chromosome conformation Hi-C data. Manual assembly curation corrected 23 missing/ misjoins, reducing the assembly length by $0.001 \%$ and the scaffold number by $31.1 \%$, and increasing the scaffold N50 by $38.9 \%$. The final assembly has a total length of $296 \mathrm{Mb}$ in 43 sequence scaffolds with a scaffold N50 of $17 \mathrm{Mb}$ (Table 1). Of the assembly sequence, $88.9 \%$ was assigned to 18 chromosomal-level scaffolds (numbered by sequence length) (Figure 1-Figure 4; Table 2). The assembly has a BUSCO (Simão et al., 2015) v5.1.2 completeness of $97.5 \%$ using the hymenoptera_odb10 reference set. While not fully phased, the assembly deposited is of one haplotype. Contigs corresponding to the second haplotype have also been deposited.

\section{Methods}

A single female $B$. hortorum was collected using a net from Wytham Woods, Oxfordshire, UK (latitude 51.77, longitude -1.339) by Liam Crowley, University of Oxford. The specimen was
Table 1. Genome data for Bombus hortorum, iyBomHort1.1.

\begin{tabular}{|c|c|}
\hline \multicolumn{2}{|l|}{ Project accession data } \\
\hline Assembly identifier & iyBomHort1 \\
\hline Species & Bombus hortorum \\
\hline Specimen & iyBomHort1 \\
\hline NCBI taxonomy ID & NCBI:txid85660 \\
\hline BioProject & PRJEB43539 \\
\hline BioSample ID & SAMEA7520483 \\
\hline Isolate information & Female, head/thorax/abdomen \\
\hline \multicolumn{2}{|l|}{ Raw data accessions } \\
\hline PacificBiosciences SEQUEL II & $\begin{array}{l}\text { ERR6054540-ERR6054544, } \\
\text { ERR6548407 }\end{array}$ \\
\hline 10X Genomics Illumina & ERR6054540-ERR6054543 \\
\hline Hi-C Illumina & ERR6054544 \\
\hline RNAseq PolyA Illumina & ERR6001535 \\
\hline \multicolumn{2}{|l|}{ Genome assembly } \\
\hline Assembly accession & GCA_905332935.1 \\
\hline Accession of alternate haplotype & GCA_905333095.1 \\
\hline Span (Mb) & 296 \\
\hline Number of contigs & 73 \\
\hline Contig N50 length (Mb) & 11 \\
\hline Number of scaffolds & 43 \\
\hline Scaffold N50 length (Mb) & 17 \\
\hline Longest scaffold (Mb) & 22 \\
\hline BUSCO* genome score & $\begin{array}{l}\text { C:97.5\%[S:97.3\%,D:0.3\%],F:0.5 } \\
\%, M: 2.0 \%, n: 5991\end{array}$ \\
\hline
\end{tabular}

*BUSCO scores based on the hymenoptera_odb10 BUSCO set using v5.1.2. $\mathrm{C}=$ complete $[\mathrm{S}=$ single copy, $\mathrm{D}=$ duplicated], $\mathrm{F}=$ fragmented, $\mathrm{M}=$ missing, $\mathrm{n}=$ number of orthologues in comparison. A full set of BUSCO scores is available at https://blobtoolkit.genomehubs.org/view/iyBomHort1.1/ dataset/CAJOSO01/busco.

snap-frozen in dry ice using a CoolRack before transferring to the Wellcome Sanger Institute (WSI) for genome sequencing and assembly.

DNA was extracted at the WSI Scientific Operations core from the head and thorax using the Qiagen MagAttract HMW DNA kit, according to the manufacturer's instructions. RNA was extracted from abdomen tissue in the Tree of Life Laboratory at the WSI using TRIzol (Invitrogen), according to the manufacturer's instructions. RNA was then eluted in $50 \mu \mathrm{l}$ 


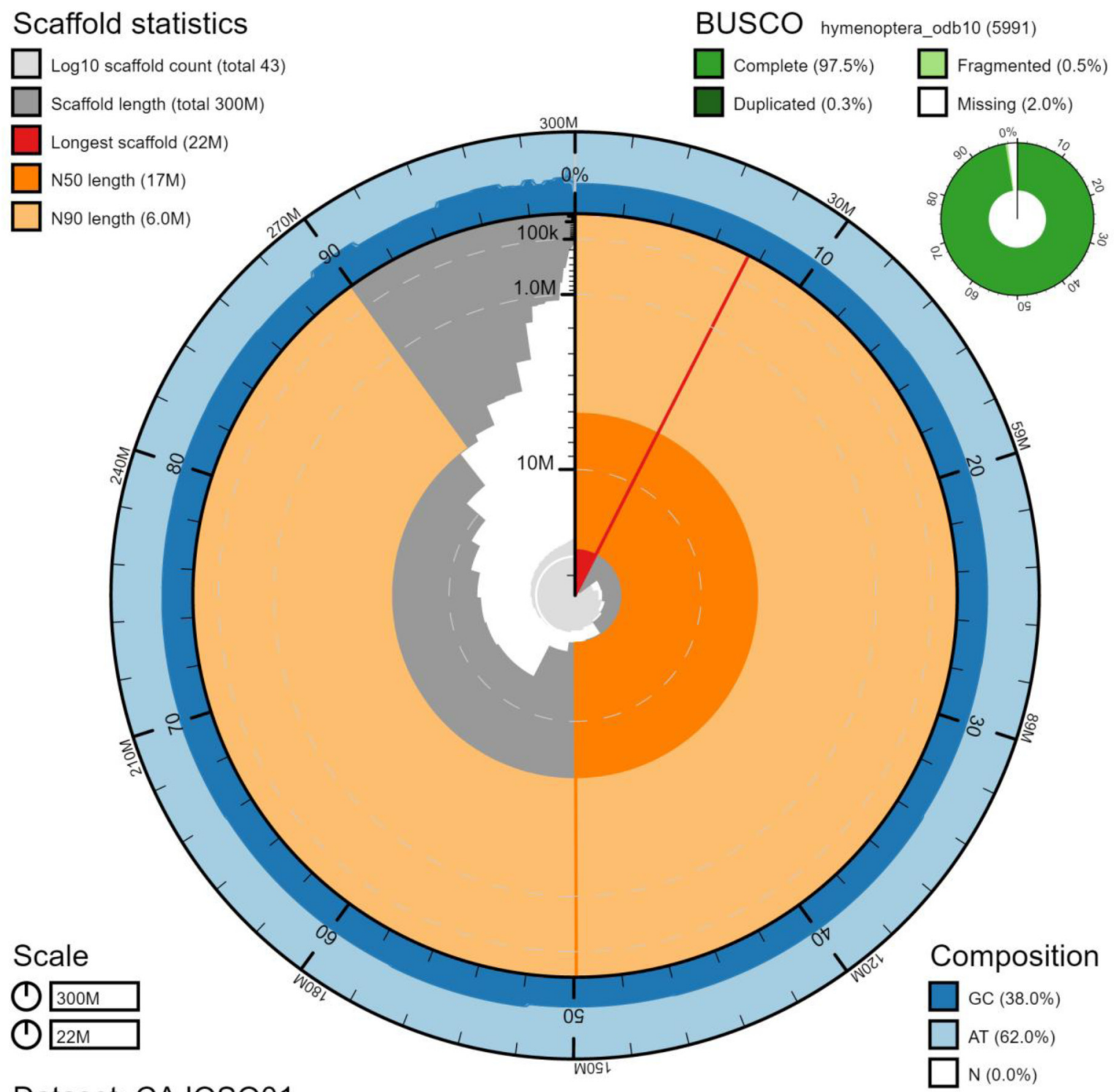

\section{Dataset: CAJOSO01}

Figure 1. Genome assembly of Bombus hortorum, iyBomHort1.1: metrics. The BlobToolKit Snailplot shows N50 metrics and BUSCO gene completeness. An interactive version of this figure is available at https://blobtoolkit.genomehubs.org/view/iyBomHort1.1/dataset/ CAJOSO01/snail.

RNAse-free water and its concentration RNA assessed using a Nanodrop spectrophotometer and Qubit Fluorometer using the Qubit RNA Broad-Range (BR) Assay kit. Analysis of the integrity of the RNA was done using Agilent RNA 6000 Pico Kit and Eukaryotic Total RNA assay.

Pacific Biosciences HiFi circular consensus and 10X Genomics read cloud sequencing libraries, in addition to PolyA RNA-Seq libraries, were constructed according to the manufacturers' instructions. Sequencing was performed by the
Scientific Operations core at the Wellcome Sanger Institute on Pacific Biosciences SEQUEL II (HiFi), Illumina HiSeq X (10X) and Illumina HiSeq 4000 (RNA-Seq) instruments. Hi$\mathrm{C}$ data were generated from head and thorax tissue using the Arima v2.0 kit and sequenced on HiSeq X.

Assembly was carried out with Hifiasm (Cheng et al., 2021). Haplotypic duplication was identified and removed with purge_dups (Guan et al., 2020). The assembly was polished with the 10X Genomics Illumina data by aligning to the 


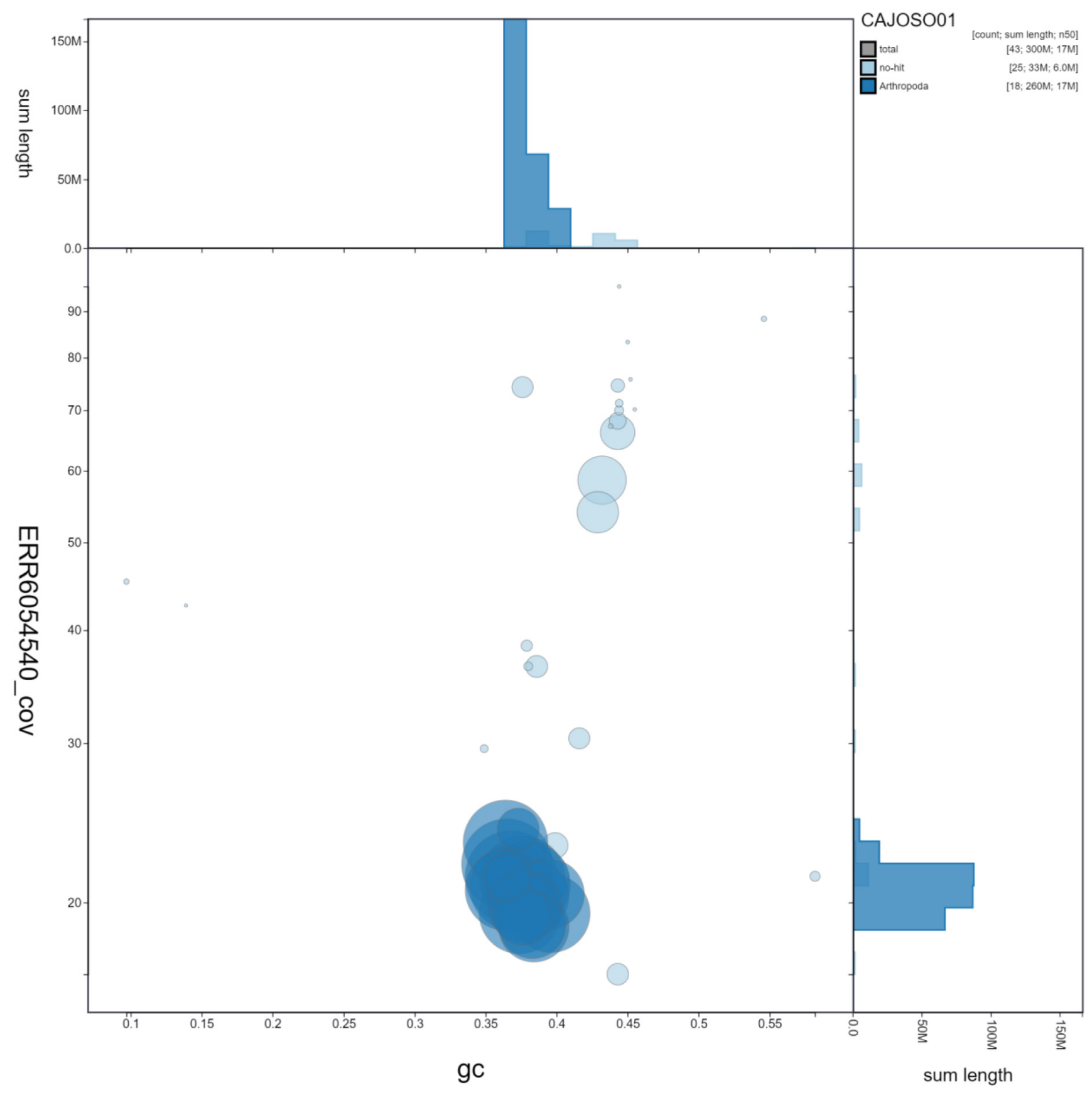

Figure 2. Genome assembly of Bombus hortorum, iyBomHort1.1: GC coverage. BlobToolKit GC-coverage plot. Scaffolds are coloured by phylum. Circles are sized in proportion to scaffold length. Histograms show the distribution of scaffold length sum along each axis. An interactive version of this figure is available at https://blobtoolkit.genomehubs.org/view/iyBomHort1.1/dataset/CAJOSO01/blob.

assembly with longranger align, calling variants with freebayes (Garrison \& Marth, 2012). One round of the Illumina polishing was applied. Scaffolding with Hi-C data (Rao et al., 2014) was carried out with SALSA2 (Ghurye et al., 2019). The assembly was checked for contamination and corrected using the gEVAL system (Chow et al., 2016) as described previously (Howe et al., 2021). Manual curation was performed using gEVAL, HiGlass (Kerpedjiev et al., 2018) and Pretext. The mitochondrial genome was assembled using MitoHiFi (Uliano-Silva et al., 2021). The genome was analysed and BUSCO scores 


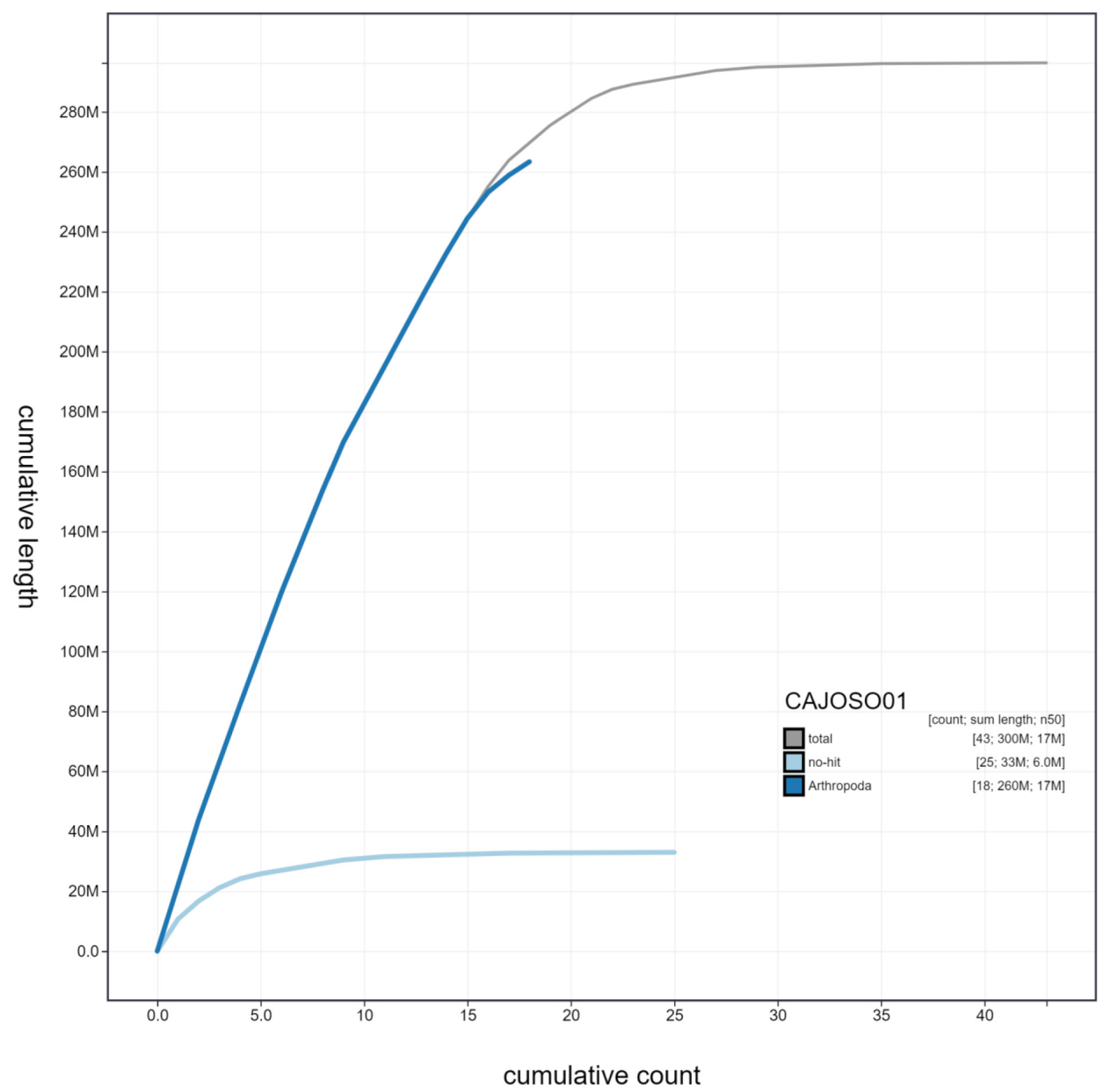

Figure 3. Genome assembly of Bombus hortorum, iyBomHort1.1: cumulative sequence. BlobToolKit cumulative sequence plot. The grey line shows cumulative length for all chromosomes. Coloured lines show cumulative lengths of chromosomes assigned to each phylum using the buscogenes taxrule. An interactive version of this figure is available at https://blobtoolkit.genomehubs.org/view/iyBomHort1.1/ dataset/CAJOSO01/cumulative.

generated within the BlobToolKit environment (Challis et al., 2020). Table 3 contains a list of all software tool versions used, where appropriate.

The materials that have contributed to this genome note have been supplied by a Darwin Tree of Life Partner. The submission of materials by a Darwin Tree of Life Partner is subject to the Darwin Tree of Life Project Sampling Code of Practice. By agreeing with and signing up to the Sampling Code of Practice, the Darwin Tree of Life Partner agrees they will meet the legal and ethical requirements and standards set out within this document in respect of all samples acquired for, and 


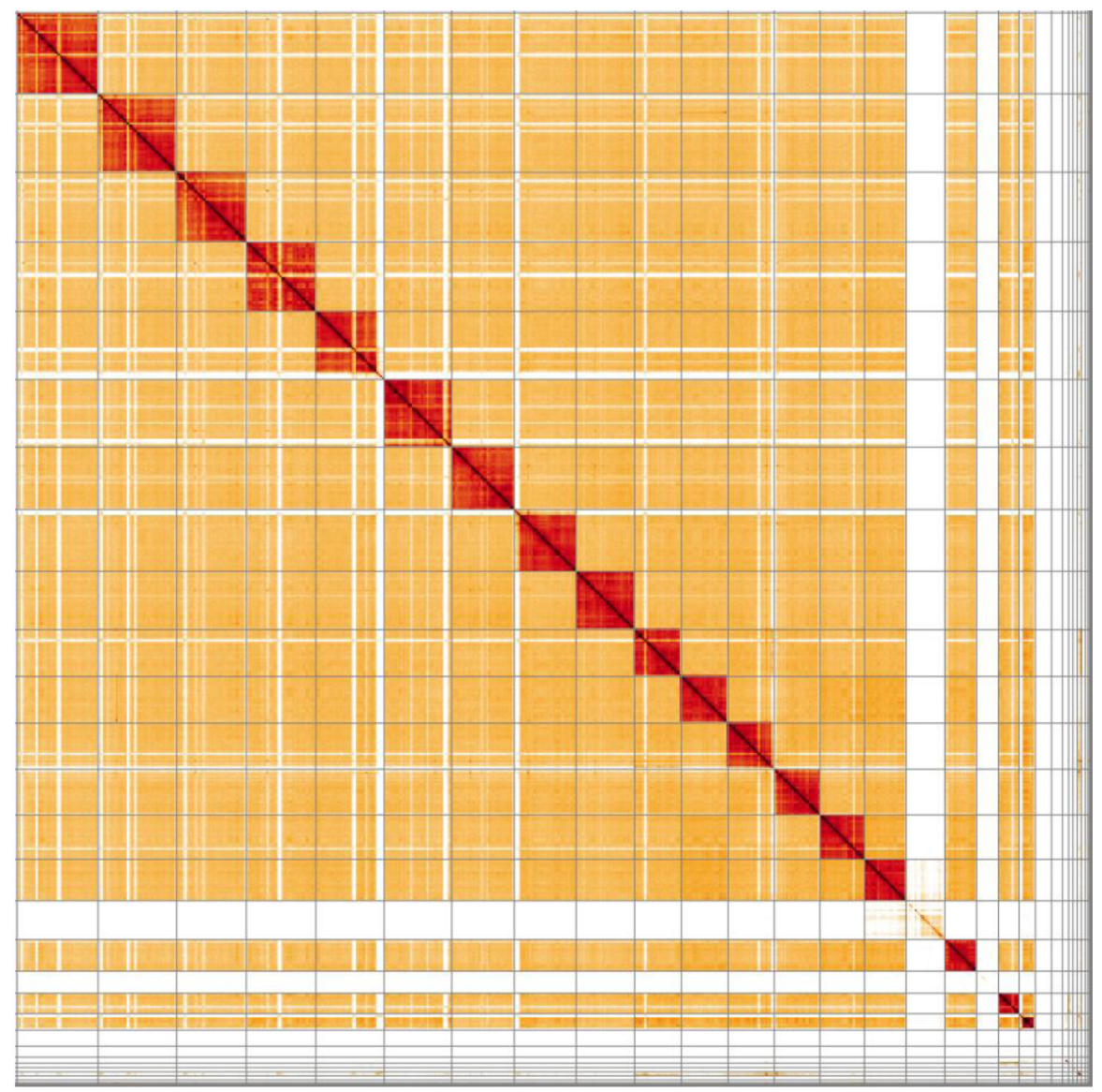

Figure 4. Genome assembly of Bombus hortorum, iyBomHort1.1: Hi-C contact map. Hi-C contact map of the iyBomHort1.1 assembly, visualised in HiGlass.

\begin{tabular}{|c|c|c|c|}
\hline \multicolumn{4}{|c|}{$\begin{array}{l}\text { Table 2. Chromosomal pseudomolecules in the } \\
\text { genome assembly of Bombus hortorum, iyBomHort1.1. }\end{array}$} \\
\hline INSDC accession & Chromosome & Size (Mb) & GC\% \\
\hline HG995188.1 & 1 & 22.27 & 36.8 \\
\hline HG995189.1 & 2 & 21.59 & 36.5 \\
\hline HG995190.1 & 3 & 19.19 & 37.6 \\
\hline HG995191.1 & 4 & 19.11 & 36.9 \\
\hline HG995192.1 & 5 & 18.77 & 36.4 \\
\hline HG995193.1 & 6 & 18.59 & 38 \\
\hline HG995194.1 & 7 & 17.19 & 37.4 \\
\hline HG995195.1 & 8 & 17.02 & 36.4 \\
\hline HG995196.1 & 9 & 16.08 & 39.6 \\
\hline
\end{tabular}

\begin{tabular}{|c|c|c|c|}
\hline INSDC accession & Chromosome & Size (Mb) & GC\% \\
\hline HG995197.1 & 10 & 12.86 & 38.4 \\
\hline HG995198.1 & 11 & 12.85 & 38.4 \\
\hline HG995199.1 & 12 & 12.68 & 39.5 \\
\hline HG995200.1 & 13 & 12.56 & 37.9 \\
\hline HG995201.1 & 14 & 12.25 & 37.6 \\
\hline HG995202.1 & 15 & 11.45 & 38.3 \\
\hline HG995203.1 & 16 & 8.71 & 37.6 \\
\hline HG995204.1 & 17 & 5.65 & 36.5 \\
\hline HG995205.1 & 18 & 4.51 & 37.3 \\
\hline HG995206.1 & MT & 0.02 & 14.2 \\
\hline- & Unplaced & 32.96 & 41.3 \\
\hline
\end{tabular}




\section{Table 3. Software tools used.}

\begin{tabular}{|l|l|l|}
\hline Software tool & Version & Source \\
\hline Hifiasm & 0.12 & Cheng et al., 2021 \\
\hline purge_dups & 1.2 .3 & Guan et al., 2020 \\
\hline SALSA2 & 2.2 & Ghurye et al., 2019 \\
\hline longranger align & 2.2 .2 & $\begin{array}{l}\text { https://support.10xgenomics.com/genome-exome/ } \\
\text { software/pipelines/latest/advanced/other-pipelines }\end{array}$ \\
\hline freebayes & $1.3 .1-17$-gaa2ace8 & Garrison \& Marth, 2012 \\
\hline MitoHiFi & 1.0 & Uliano-Silva et al., 2021 \\
\hline gEVAL & N/A & Chow et al., 2016 \\
\hline HiGlass & 1.11 .6 & Kerpedjiev et al., 2018 \\
\hline PretextView & $0.1 . x$ & https://github.com/wtsi-hpag/PretextView \\
\hline BlobToolKit & 2.6 & Challis et al., 2020 \\
\hline
\end{tabular}

supplied to, the Darwin Tree of Life Project. Each transfer of samples is further undertaken according to a Research Collaboration Agreement or Material Transfer Agreement entered into by the Darwin Tree of Life Partner, Genome Research Limited (operating as the Wellcome Sanger Institute), and in some circumstances other Darwin Tree of Life collaborators.

\section{Data availability}

European Nucleotide Archive: Bombus hortorum (garden bumblebee). Accession number PRJEB43539: https://identifiers. org/ena.embl:PRJEB43539

The genome sequence is released openly for reuse. The $B$. hortorum genome sequencing initiative is part of the Darwin Tree of Life (DToL) project. All raw sequence data and the assembly have been deposited in INSDC databases. The genome will be annotated using the RNA-Seq data and presented through the Ensembl pipeline at the European Bioinformatics Institute. Raw data and assembly accession identifiers are reported in Table 1.

\section{Acknowledgments}

Members of the University of Oxford and Wytham Woods Genome Acquisition Lab are listed here: https://doi.org/10.5281/ zenodo.4789929.

Members of the Darwin Tree of Life Barcoding collective are listed here: https://doi.org/10.5281/zenodo.4893704.

Members of the Wellcome Sanger Institute Tree of Life programme collective are listed here: https://doi.org/10.5281/ zenodo. 5377053 .

Members of Wellcome Sanger Institute Scientific Operations: DNA Pipelines collective are listed here: https://doi.org/10.5281/ zenodo.4790456.

Members of the Tree of Life Core Informatics collective are listed here: https://doi.org/10.5281/zenodo.5013542.

Members of the Darwin Tree of Life Consortium are listed here: https://doi.org/10.5281/zenodo.4783559.
Brown B, Scott RR, Macfarlane RP: An Assessment of Vicia Faba and Trifolium Pratense as Forage Crops for Bombus Hortorum. N Z Entomol. 1992; 15(1): 42-47.

Publisher Full Text

Challis R, Richards E, Rajan J, et al.: BlobToolKit - Interactive Quality Assessment of Genome Assemblies. G3 (Bethesda). 2020; 10(4): 1361-74. PubMed Abstract | Publisher Full Text | Free Full Text

Cheng $\mathrm{H}$, Concepcion GT, Feng X, et al.: Haplotype-Resolved de Novo Assembly Using Phased Assembly Graphs with Hifiasm. Nat Methods. 2021 18(2): 170-75.

PubMed Abstract | Publisher Full Text | Free Full Text
Chow W, Brugger K, Caccamo M, et al.: gEVAL - a Web-Based Browser for Evaluating Genome Assemblies. Bioinformatics. 2016; 32(16): 2508-10. PubMed Abstract | Publisher Full Text | Free Full Text

Edwards M, Jenner M: Field Guide to the Bumblebees of Great Britain \& Ireland. Ocelli Ltd, 2005

Reference Source

Garrison E, Marth G: Haplotype-Based Variant Detection from Short-Read Sequencing. arXiv:1207.3907. 2012.

Reference Source

Ghurye J, Rhie A, Walenz BP, et al.: Integrating Hi-C Links with Assembly Graphs for Chromosome-Scale Assembly. PLoS Comput Biol. 2019; 15(8): 
e1007273.

PubMed Abstract | Publisher Full Text | Free Full Text

Guan D, McCarthy SA, Wood J, et al.: Identifying and Removing Haplotypic Duplication in Primary Genome Assemblies. Bioinformatics. 2020; 36(9): 2896-98.

PubMed Abstract | Publisher Full Text | Free Full Text

Howe K, Chow W, Collins J, et al.: Significantly Improving the Quality of Genome Assemblies through Curation. GigaScience. 2021; 10(1): giaa153. PubMed Abstract | Publisher Full Text | Free Full Text

Kells AR, Goulson D: Preferred Nesting Sites of Bumblebee Queens (Hymenoptera: Apidae) in Agroecosystems in the UK. Biol Conserv. 2003; 109(2): 165-74.

Publisher Full Text

Kerpedjiev P, Abdennur N, Lekschas F, et al.: HiGlass: Web-Based Visual
Exploration and Analysis of Genome Interaction Maps. Genome Biol. 2018; 19(1): 125.

PubMed Abstract | Publisher Full Text | Free Full Text

Rao SS, Huntley MH, Durand NC, et al:: A 3D Map of the Human Genome at Kilobase Resolution Reveals Principles of Chromatin Looping. Cell. 2014; 159(7): 1665-80.

PubMed Abstract | Publisher Full Text | Free Full Text

Simão FA, Waterhouse RM, Ioannidis P, et al.: BUSCO: Assessing Genome Assembly and Annotation Completeness with Single-Copy Orthologs.

Bioinformatics. 2015; 31(19): 3210-12.

PubMed Abstract | Publisher Full Text

Uliano-Silva M, Nunes JGF, Krasheninnikova K, et al.: marcelauliano/MitoHiFi: mitohifi_v2.0. 2021.

Publisher Full Text 


\section{Open Peer Review}

\section{Current Peer Review Status:}

\section{Version 1}

Reviewer Report 29 October 2021

https://doi.org/10.21956/wellcomeopenres.18990.r46472

(C) 2021 Brown S. This is an open access peer review report distributed under the terms of the Creative Commons Attribution License, which permits unrestricted use, distribution, and reproduction in any medium, provided the original work is properly cited.

\section{Susan Brown}

KSU Bioinformatics Center, Division of Biology, Kansas State University, Manhattan, KS, USA

This report on the genome assembly for the garden bumblebee is concise, complete and clearly written. As with all Tree of LIfe projects, DNA from a single female individual was sequenced using PacBio long read and scaffolding with $\mathrm{Hi}-\mathrm{C}$ reads. The Blobtools kit provides an easily understandable graphic summary of the assembly. The BUSCO score of $97.5 \%$ indicates a predominantly complete genome representation. This assembly is in excellent shape for annotation and downstream analysis.

Is the rationale for creating the dataset(s) clearly described?

Yes

Are the protocols appropriate and is the work technically sound?

Yes

Are sufficient details of methods and materials provided to allow replication by others? Yes

Are the datasets clearly presented in a useable and accessible format?

Yes

Competing Interests: No competing interests were disclosed.

Reviewer Expertise: Genomics, bioinformatics and Deelopmental Genetics of the red flour beetle.

I confirm that I have read this submission and believe that I have an appropriate level of expertise to confirm that it is of an acceptable scientific standard. 


\section{https://doi.org/10.21956/wellcomeopenres.18990.r46468}

(C) 2021 Waterhouse R. This is an open access peer review report distributed under the terms of the Creative Commons Attribution License, which permits unrestricted use, distribution, and reproduction in any medium, provided the original work is properly cited.

\section{Robert M. Waterhouse}

Department of Ecology and Evolution, University of Lausanne, Lausanne, Switzerland

This Data Note presents a clear and comprehensive description of all the steps taken to generate the Bombus hortorum genome assembly spanning $296 \mathrm{Mb}$ with $90 \%$ assigned to 18 chromosomallevel scaffolds. As a member of an important group of pollinators, the rationale for building these resources is clear. The described data collection and analysis methods follow the best practices in the field and have delivered a high-quality complete and accurate chromosome-level reference genome.

Is the rationale for creating the dataset(s) clearly described?

Yes

Are the protocols appropriate and is the work technically sound?

Yes

Are sufficient details of methods and materials provided to allow replication by others? Yes

Are the datasets clearly presented in a useable and accessible format? Yes

Competing Interests: No competing interests were disclosed.

Reviewer Expertise: Arthropod genomics

I confirm that I have read this submission and believe that I have an appropriate level of expertise to confirm that it is of an acceptable scientific standard.

Reviewer Report 19 October 2021

https://doi.org/10.21956/wellcomeopenres.18990.r46470

(C) 2021 Richards S. This is an open access peer review report distributed under the terms of the Creative Commons Attribution License, which permits unrestricted use, distribution, and reproduction in any medium, provided the original work is properly cited.

\section{Stephen Richards}

Human Genome Sequencing Center, Department of Human and Molecular Genetics, Baylor College of Medicine, Houston, TX, USA 
The authors present a truly excellent extremely high-quality genome reference of Bombus hortorum. The reference genome is high quality enough that it will stand the test of time, and likely be the go-to reference for at least the next 100 years, and possibly much longer. In terms of data access, I was able to easily find and download the sequence from my INSDC database (NCBI) and had no trouble blasting my favorite gene against it (something that not all genome data providers always do, and so to be congratulated here.). Not really sure there is anything else I can add for a review of a data note, the data has been very well generated in a transparent manner and very well made globally available.

Is the rationale for creating the dataset(s) clearly described?

Yes

Are the protocols appropriate and is the work technically sound?

Yes

Are sufficient details of methods and materials provided to allow replication by others? Yes

Are the datasets clearly presented in a useable and accessible format?

Yes

Competing Interests: No competing interests were disclosed.

Reviewer Expertise: Arthropod genomics and genomics in general.

I confirm that I have read this submission and believe that I have an appropriate level of expertise to confirm that it is of an acceptable scientific standard. 